\title{
Ultrasound Prevalence of Gallbladder Disease in Hail, Saudi Arabia
}

\author{
Maram N Alawad ${ }^{1}$, Abdalaziz M Almotlaq ${ }^{2}$, Shaikhah H Alorf ${ }^{3}$, Nouf H Alshammari ${ }^{4}$, \\ Ashjan M Almhanaa ${ }^{5}$, Hanin M Aljohani ${ }^{6}$, Mohammed I Elsayed ${ }^{7}$
}

\begin{abstract}
Objective: Cholestasis is one of themost common gastrointestinal disorders requiringhospitalization. While different factors influence gallstone formation, these factors are not the same in different cultures or geographical locations. We determinedthe prevalence of gallbladder disease as assessed by ultrasonography and its complications in Hail City, Saudi Arabia. Methods: Patients who underwent emergency or elective abdominal ultrasonographyat King Khalid Hospital, the largest tertiary hospital in the Hail region of Saudi Arabia, between January 2013 and December 2013 were retrospectively analyzed. Results: Of the 4552 patients analyzed, 494(10.9\%) had gallstones. Of these 494 patients, 173 (35\%) were male, 321 (65\%) were female and 337 (68.2\%) were aged $>35$ years.Three hundred twenty-sixpatients $(66 \%)$ had multiple stones, whereas 168 patients (34\%) had a single stone. Marked and mild wall thickening were found in 180 patients (36.4\%) and 155 patients (31.4\%), respectively. Common bile duct dilatation was present in 36 patients (7.3\%), fatty liver in 106 patients (21.5\%), hepatomegaly in 36 patients (7.3\%), cirrhosis in 20 patients (4\%) and ascites in21 patients(4.3\%). Of the 494 patients, 335 (67.8\%) were symptomatic.Saudi females had the highest prevalence of gallbladder disease (60.1\%) followed by Saudi males (31.6\%), non-Saudi females (4.9\%), and non-Saudi males (3.8\%). Conclusion: The prevalence of gallbladder disease was higher in Hail City compared with other cities in Saudi Arabia.
\end{abstract}

Keywords: Ultrasound Prevalence Gallbladder Disease Hail Saudi Arabia

\section{Introduction}

Gallstone disease represents a significant burden for health care systems worldwide. Gallstones are more common in women than in men $(24 \%$ vs. $12 \%)$. Symptomatic disease occurs in $10-20 \%$ of patients. Gallstones can occur anywhere within the biliary tree. Causes of gallstone formation include active bile saturation, cholesterol precipitation, crystal formation, impaired gallbladder function, and diminished intrahepatic circulation of bile acid. Gallstones are of three types, cholesterol stones, mixed stones, and pigment stones. Mixed stones are the most common (80\%) followed by cholesterol stones $(15 \%)$ and pigment stones $(5 \%)$.

Ultrasound is the most sensitive and specificdiagnostic test in suspected gallstone or biliary disease, such asbiliary lithiasis. Diagnostic ultrasound is also advantageous in terms of its noninvasiveness and cost effectiveness. Although the diagnostic sensitivity of ultrasound is operator dependent,generally this method it is highly specific and sensitive $(>95 \%)$. On ultrasound, thestone-filled gallbladder demonstrates a wall-echo-shadow sign. The prevalence of gallstone disease varies according to geographical location.The averageprevalence of gallstone disease in Western countries is estimated to be more than $10 \%$.More than $15 \%$ of the North American population suffers from gallstone disease.In Asian countries, the prevalence of gallstone disease is approximately $10 \%$, whereas the rate in African countriesis less than 5\%. On the other hand, the average prevalence in Middle Eastern countries ranges from $4-12 \%$. The increasein prevalence may be due to lifestyle and dietary (high-energy diet consumption) modificationsover the last few years. Epidemiological investigation of gallstone disease is important for prevention and improvement of diagnostic and therapeuticstrategies.

The objective of this study was to evaluate the prevalence of gallstone disease in Hail, Saudi Arabia.
Hail is located in the northernregion of Saudi Arabia and has an area of $103887 \mathrm{~km}$ and a population of 527000 (2004).

\section{Methods}

Patients who underwent emergency or elective abdominal ultrasonography at King Khalid Hospital (Hail City, Saudi Arabia) between January 2013 and December 2013 were retrospectively analyzed. Patients consented to the review of their medical records and abdominal ultrasound reports for research purposes. Medical records and reports were deidentified prior to review. All information obtained from patient medical records was kept confidential. Patients could refuse to participate at any time during the data collection period. Patients were not compensated for their participation.

Initially, all patients were scanned in the supine position. The gallbladder was examined in at least 2 planes and 2 positions to obtain satisfactory visualization.Ultrasound diagnoses of acute and chronic cholcystitis were made based on the following findings: wall thickening (chronic, $>3 \mathrm{~mm}$ ), mucosal irregularities, calculus within the gallbladder lumen, and positive Murphy's sign (tenderness when the transducer is pressed on the gall bladder).After diagnosis, the number of stones, wall thickness, common bile duct (CBD) diameter, and presence of fatty liver,hepatomegaly, and ascites were noted in each case. Gallstones were classified as symptomatic or asymptomatic based on wall thickness and patient symptoms.All data were collected,tabulatedand analyzed at the end of the study.

Statistical Analysis SPSS used

\section{Results}

The records of 4552 patients who underwent ultrasonographybetween January 2013 and December 2013 were retrospectively reviewed and analyzed.Of these 4552 


\section{International Journal of Science and Research (IJSR) \\ ISSN (Online): 2319-7064}

Index Copernicus Value (2013): 6.14 | Impact Factor (2014): 5.611

patients, 494 patients had gallstones (10.9\%). Of the494 patients with gallstones, $173(35 \%)$ were males, 321 (65\%) were females, and $337(68.2 \%)$ were aged $>35$ years. Three hundred twenty-six patients $(66 \%)$ had multiple stones, whereas 168 patients (34\%) had a single stone. Most patients $(335,67.8 \%)$ had wall thickening. CBD dilatation was present in 36 patients (7.3\%).Fatty liver, hepatomegaly,liver cirrhosis, and ascites were present in 100 $(21.5 \%), 36(7.3 \%), 20(4 \%)$, and $21(4.3 \%)$ patients, respectively.

We further analyzed the prevalence of gallbladder disease according to gender and nationality. Saudi females had the highest incidence of gallbladder disease $(297 / 494,60.1 \%)$ followed by Saudi males (156/494, 31.6\%),non-Saudi females were 24 patients $(4.9 \%)$, and non-Saudi males were 17 patients (3.8\%). Of the Saudi patients, 106 male patients (31.4\%) and 200 female patients $(59.3 \%)$ were aged 35 years or older. Eight-four male patients $(25.7 \%)$ and 206 female patients $(63.2 \%)$ had multiple stones, whereas 59 male patients $(35.1 \%)$ and 100 female patients $(59.5 \%)$ had a single stone. CBD dilatation was present in 8 male patients $(22.2 \%)$ and23 female patients $(63.9 \%)$, wall thickening in 101 male patients $(30.1 \%)$ and 200 female patients $(59.7 \%)$, fatty liver in 29 male patients $(27.4 \%)$ and in 60 female patients $(56.6 \%)$, ascites in 9 male patients $(42.9 \%)$ and 11 female patients $(52.4 \%)$, hepatomegaly in 5 male patients $(13.9 \%)$ and 25 female patients $(69.4 \%)$, and cirrhosis in 10 male patients $(50 \%)$ and 10 female patients $(50 \%)$.

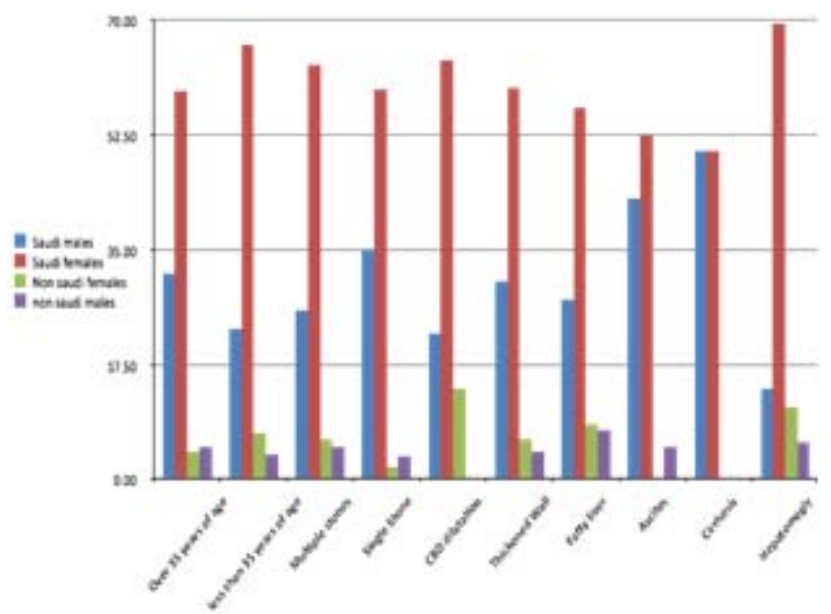

Figure 1: Prevalence of gallbladder disease in Hail city

\section{Discussion}

Gallstones are a major cause of hospital admission,morbidity and mortality, particularly in developing countries.Gallstones account for more than 95\% of biliary tract disorders. This study evaluated the prevalence of gallbladder disease among Saudi and non-Saudi patients in the Hail region of Saudi Arabia, with the aim of furthering our understanding of the epidemiology of gallstone diseasein this region. Furthermore, we analyzed stone number, wall thickness and complications according to gender and nationality. Theworldwide prevalence of gallstone disease as assessed by ultrasonography is reported to be between $5.9-21.9 \%$. This variabilityin gallstone prevalence may be related to differences in diet and lifestyle. Gallstones can occur at any age; however, the incidence increases with age and is most prevalent in the $4^{\text {th }}$ and $5^{\text {th }}$ decades of life. Studies have shown that gallstones are more common among females than males. Most of these studies have been performed at hospitals in Western countries.

We analyzed the number of gallbladder calculi at the time of presentation.Gallstone type (cholesterol/mixed/pigment stones) was not determined and was a limitation of our study.Although chemical and spectrophotometric analysis of postoperative specimens would have been ideal, this was beyond the scope of our study.

In conclusion, gallstone disease is a major cause of morbidity in Saudi Arabia. Real time ultrasonography is a useful tool to assess gallstonedisease burden in individual communities. The female population of Saudi Arabia was found to have a higher risk of developing gallstone disease at an earlier age compared to with the male population.

\section{References}

[1] Zamani F, Sohrabi M, Alipour A, Motamed N, Saeedian FS, Pirzad R, et al. Prevalence and risk factors of cholelithiasis in Amol city, northern Iran: A population based study. Arch Iran Med 2014;17:750-754.

[2] Chen CH, Huang MH, Yang JC, Nien CK, Etheredge GD, Yang CC, et al. Prevalence and risk factors of gallstone disease in an adult popula- tion of Taiwan: an epidemiological survey. J GastroenterolHepatol2006; 21:1737-1743.

[3] Central Department of Statistics. Population growth rate among the population in 2004 to 2010 censuses. Riyadh (SA): Ministry of Economy and Planning, Saudi Arabia; 2010.

[4] Murshid KR. Syptomatic gallstones: a disease of young Saudi women. Saudi J Gastroenter 1998;4:159-162.

[5] Mokhtar AMA. Cholelithiasis in the western region of Saudi Arabia. East African Medical J 1990;67:286-290.

[6] Ahmed AF, El-Hassan OM, Mahmoud ME. Risk factors for gallstone formation in young Saudi women: a case control study. Ann Saudi Med 1992;12:395-399.

[7] Khan HN, Harrison M, Bassett EE, Bates T. A 10-year follow-up of a longitudinalstudy of gallstone prevalence at necropsy in South East England. Dig Dis Sci 2009; 54: 2736-2741.

[8] Bainton D, Davies GT, Evans KT, Gravelle IH. Gallbladder disease. Prevalence in a South Wales industrial town. N Engl J Med1976 May 20;294:11471149.

[9] Marschall HU, Einarsson C. Gallstone disease. J Intern Med 2007; 261:529- 542 . 